\title{
Attempt Suicide by Inserting a Ball-Pen into the Brain: A Case Report
}

\author{
Chi-Man Yip \\ Division of Neurosurgery, Kaohsiung Veterans General Hospital, Kaohsiung, Chinese Taipei \\ Email: yip_chiman@yahoo.com
}

Received February 19, 2012; revised March 9, 2012; accepted March 17, 2012

\begin{abstract}
Purpose: Closed head injuries are more common than penetrating head injuries in our clinical practices. Penetrating head injuries can be happened in industrial accidents, car accidents, fall accidents or criminal activities, also they can be occurred in self-inflicted injuries. We reported a case of self-inflicted penetrating head injury to highlight the importance of suicide precaution and review the principle of management of penetrating head injury. Methods and Results: A 58 year-old woman, who has hypertension and major depression, attempted suicide by inserting a ball-pen into her brain through the right temple in the psychiatric ward. CT of brain showed a foreign body penetrating through the right sphenoid ridge to intracranial right frontal-temporal junction, right parasella and suprasella cistern to interpeduncular cistern and acute subarachnoid hemorrhage in the basal cistern and right sylvian fissure. She underwent an emergent neurosurgical procedure to remove the ball-pen, to evacuate the intracranial hematoma and to repair the lacerated dura. She recovered gradually and was clear after surgery, but a right oculomotor palsy and very mild left hemiparesis were left. Discussion: We learn a lesson from this patient, even a ball-pen can become a weapon in the hands of depression patients. All clinicians should be aware of the psychological condition of each patient and suicide precaution should be kept in mind in clinical practice.
\end{abstract}

Keywords: Ball-Pen; Major Depression; Penetrating Head Injury; Self-Inflicted Injury

\section{Introduction}

Self-inflicted injuries among psychiatric patients are not uncommon. Ball-pen is a very common stationery, however, it can become a murderous tool. We report a rare case of a 58 -year-old woman who committed suicide by inserting a ball-pen into her brain through the right sphenoid ridge of skull and got a severe penetrating brain injury. To our knowledge, there were only 3 reports of self-introducing a ball-pen intracranially written in the English literatures [1-3]. All of these three patients had history of psychiatric diseases and they passed the ballpen into the brain either through the paranasal sinus or orbit which are thin bones. In contrast, our patient introduced the ball-pen intracranially through the sphenoid ridge which is very thick and strong.

\section{Case Report}

A 58-year-old woman with hypertension and depression admitted to our psychiatric ward. During the hospital stay, on one day, after the lunch, she got a ball-pen from another patient and inserted it into her brain through her right temple in a toilet. Because she stayed in the toilet for a long time, so the nursing staff opened the toilet door and found her sitting on the floor with a ball-pen penetrating into her right side of head almost in $2 / 3$ of the whole length (Figure 1). She was unconscious with anisocoric pupils, besides the open wound on her head, multiple shallow wounds were found on her neck, her right palm and her left wrist. After resuscitation, a brain com-

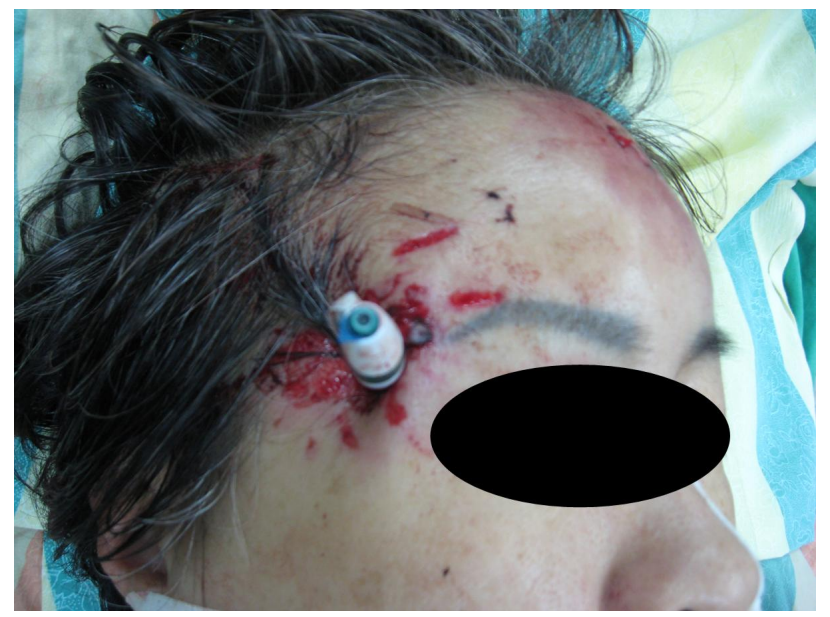

Figure 1. A ball-pen penetrating into the right side of the patient's head. 
puted tomography (CT) scan was done showing a foreign body penetrating through the right sphenoid ridge to intracranial right fronto-temporal junction, right parasella and suprasella cistern, even deep to interpeduncular cistern and acute subarachnoid hemorrhage in the basal cistern and right sylvian fissure without the right orbital injury (Figure 2).

Under general anesthesia, she underwent an emergent right pterional craniotomy with the removal of the ballpen following the original entrance trajectory, evacuation of the traumatic subarachnoid hematoma, repairing of the lacerated dura and wound debridement. Broad-spectrum antibiotics was administered for a prolonged period to prevent meningitis and brain abscess formation. After surgery, she recovered gradually and regained her consciousness; she proved that the injury was self-inflicted. Her post-operative course was uneventful. Rehabilitation program was arranged after her surgical condition was stable, and psychiatric physicians kept on treatment to her depression. At discharge, she had right oculomotor palsy and very mild left hemiparesis which probably due to direct trauma to her right oculomotor nerve and her right cerebral peduncle as the ball-pen passing. The muscle power of her left upper and left lower limbs was grade 5-. Her post-operative brain CT showed neither intracranial hematoma nor brain abscess.

\section{Discussion}

Cranial penetrating injuries due to foreign bodies other than bullets are not frequent. These injuries can be happened in industrial accidents, car accidents, fall accidents or criminal activities [4,5]. From literatures, some of these injuries occurred in psychiatric patients attempting suicide and the causative objects described in the clinical reports include nail, pencil, ball-pen, toothbrush, harpoon, spectacle arm, screw, ice pick, chopsticks, metallic wires, sewing needles [1-4,6-9]. To date, there are only 3 English literatures reporting self-inflicted penetrating brain injury with a ball-pen; in all of these reports, the ballpens got into the brain either passing through the orbit or

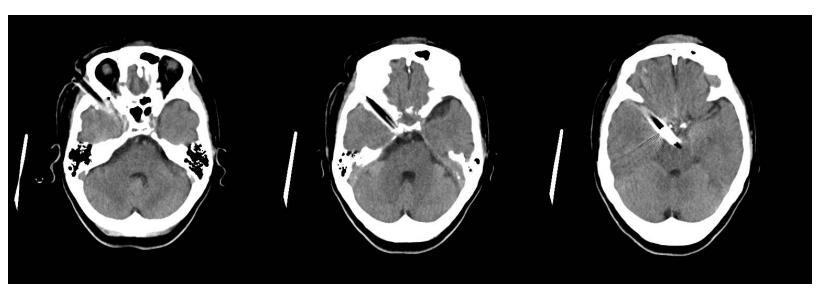

Figure 2. Consecutive axial slices of brain CT showed a foreign body penetrating through the right sphenoid ridge to intracranial right fronto-temporal junction, right parasella and suprasella cistern, even deep to inter-peduncular cistern and acute subarachnoid hemorrhage in the basal cistern and right sylvian fissure. the nasal cavity and paranasal sinus which are thin and delicate bones [1-3]. In our patient, the ball-pen passed through the right sphenoid ridge which was thick and tough implying how large the force she applied to the ball-pen. From this patient, we learn that all objects can be a weapon in the hands of patients with psychiatric disorders. All clinicians should be aware of the psychological condition of each patient and suicide precaution should be kept in mind in clinical practice.

Penetrating brain injury can result with major vascular injury, brain parenchymal damage, CSF leakage, seizures, meningitis and brain abscess formation, causing severe morbidity and even mortality $[1,3-5,7]$. Brain CT scan is the best tool of evaluation of penetrating brain injuries because it can localize the foreign bodies, reveal fractures, intracranial air and hematoma and the extent of brain damage [4,5]. Prompt surgical intervention is mandatory to reduce the mortality and complication rate. The goals of surgery are removal of the foreign bodies and bone fragments, debridement of the path, evacuation of hematoma, and repair of vascular or dural damage $[2,4,5$, 7,9]. All self-inflicted injured patients should have a complete psychiatric evaluation and treatment is necessary when indicated $[4,8]$.

\section{Conclusion}

Suicide precaution becomes an important issue as the depression patients are increasing. Penetrating head injury need prompt surgical intervention to remove the foreign bodies, hematoma and nonviable brain tissue, to repair the broken dura, to reconstruct the skull base and vascular structures if indicated. Infection control is important in the management of penetrating head injury, therefore, a team including neurosurgeons, infection physicians, psychiatrists, rehabilitation physicians and social workers is needed to manage self-inflicted penetrating head injury patients to improve their outcome.

\section{REFERENCES}

[1] D. I. Bowen, "Self-Inflicted Orbitocranial Injury with a Plastic Ballpoint Pen," British Journal of Ophthalmology, Vol. 55, No. 6, 1971, pp. 427-430. doi:10.1136/bjo.55.6.427

[2] S. Sharif, G. Roberts and J. Phillips, "Transnasal Penetrating Brain Injury with a Ball-Pen," British Journal of Neurosurgery, Vol. 14, No. 2, 2000, pp. 159-160. doi:10.1080/02688690050004660

[3] P. Lunetta, A. Öhberg and A. Sajantila, "Suicide by Intracerebellar Ballpoint Pen," American Journal of Forensic Medicine and Pathology, Vol. 23, No. 4, 2002, pp. 334-337. doi:10.1097/00000433-200212000-00006

[4] C. Gökcek, Y. Erdem, E. Köktekir, M. Karatay, M. A. Bayar, N. Edebali, et al., "Intracranial Foreign Body," Turkish Neurosurgery, Vol. 17, No. 2, 2007, pp. 121-124. 
[5] H. L. Lin, H. C. Lee and D. Y. Cho, "Management of Transorbital Brain Injury," Journal of the Chinese Medical Association, Vol. 70, No. 1, 2007, pp. 36-38. doi:10.1016/S1726-4901(09)70299-0

[6] A. Al-Anazi, I. Al-Luwimi and M. Nasser, "Transorbital Penetrating Craniocerebral Injury by a Spectacle arm: Suicidal Attempts," British Journal of Neurosurgery, Vol. 17, No. 4, 2003, pp. 368-369. doi:10.1080/02688690310001601315

[7] B. Cemil, K. Tun, O. Yiğenoğlu and E. Kaptanoğlu, "Attempted Suicide with Screw Penetration into the Cra- nium," Turkish Journal of Trauma \& Emergency Surgery, Vol. 15, 2009, pp. 624-627.

[8] K. Kishore, S. Sahu, P. Bharti, S. Dahiya, A. Kumar and A. Agarwal, "Management of Unusual Case of SelfInflicted Penetrating Craniocerebral Injury by a Nail," Journal of Emergency, Trauma and Shock, Vol. 3, No. 2, 2010, pp. 193-196. doi:10.4103/0974-2700.62115

[9] A. Iordache, J. M. Kaya, J. R. Alliez and B. Alliez, "A Rare Case of Severe Craniocerebral Trauma with Penetrating Head Injury," Romanian Neurosurgery, Vol. 18, 2011, pp. 77-81 